only $35 \%$ are aware that undetectable viral load is associated with a decreased risk of sexual transmission. The knowledge that HIV transmission decreases with low VL is higher among women with higher level of education $(\mathrm{OR}=1.72)$, women diagnosed for at least two years $(\mathrm{OR}=1.91)$ and among discordant couples $(\mathrm{OR}=2.56)$. And, the non-use of condoms in the last sexual intercourse is higher among those who know that HIV transmission decreases with low VL and among those with $\mathrm{HIV}+$ partner $(\mathrm{OR}=1.69$ and 2.56, respectively), regardless level of schooling.

Conclusion Women can take advantage of up-to-date information on HIV prevention once they have it. Meaningful information is a critical piece to ensure a pleasurable and safe sexual life of WLHIV, free of constraints for not using condoms $100 \%$ of the time. Sexuality and its constraints are always changing. New challenges are placed in the fourth decade of the AIDS epidemic; some of them refer to gender and social inequalities in the access to information.

\section{P4.90 WHICH SEXUALLY TRANSMITTED INFECTIONS DO GAY AND BISEXUAL MEN FIND MOST SCARY AND WHY? A QUALITATIVE STUDY}

${ }^{1,2,4} \mathrm{D}$ Reid, ${ }^{1,2} \mathrm{~J}$ Datta, ${ }^{1,3,4} \mathrm{~S}$ Wayal, Cath Mercer, ${ }^{1,4} \mathrm{G}$ Hughes, ${ }^{1,2} \mathrm{P}$ Weatherburn. ${ }^{1}$ National Institute of Health Research (NIHR) Health Protection Research Unit (HPRU) in Blood Borne and Sexually Transmitted Infections, UK; ${ }^{2}$ Centre for Sexual Health and HIV, Research Department of Infection and Population Health, University College London, UK; ${ }^{3}$ London School of Hygiene and Tropical Medicine, London, UK; ${ }^{4}$ Public Health UK

\subsection{6/sextrans-2017-053264.585}

Introduction As a result of high burden of infection, men who have sex with men (MSM) are a priority for sexually transmitted infection (STI) prevention interventions including promoting regular testing. Effective intervention design requires understanding of MSM's knowledge and perceptions of STIs and their social representations which may affect attitudes and behaviour related to risk, testing and treatment. To inform STI prevention interventions and development of questions inclusion in an online survey that would link with surveillance data on testing and diagnosis we assessed MSM's knowledge and attitudes towards STIs.

Methods We recruited a diverse sample of MSM in 4 English cities, through social networking and community organisations.61 MSM attended one of eight 90 min focus group discussions. Topics included knowledge of, and attitudes towards STIs. We ran a discussion and ranking exercise in which names of 11infections, specified below, printed in large font on separate sheets were laid out and respondents were asked what they knew about each and to rank them in order of most to least 'scary', giving reasons for their ranking. Infections included: HIV, syphilis, gonorrhoea, chlamydia, HPV/genital warts, genital herpes, Shigella, scabies, hepatitis A (HAV), B (HBV) and C (HCV). Discussions were audio recorded, transcribed and thematic data analysis conducted.

Results Participants demonstrated both wide and detailed knowledge as well as lack of awareness depending on individuals and infections. While the greatest knowledge and experience was of HIV, few had heard of or knew much about Shigella. No focus groups were unanimous in their ranking of infections and patterns were not the same across groups. HIV and HCV were ranked the two most 'scary' by all groups and syphilis was among the three most scary. Herpes was also ranked highly by all but one group while scabies was similarly ranked least scary. Other infections ranked between with no clear patterning within or between groups. The exercise produced a complex range of explanations relating to fear of individual infections and of STIs in general. Participants weighed up the scary and less scary attributes of the infections depending on the extent of their knowledge about and experience of them, their prevalence among MSM, associated stigma, transmission mechanisms, contagiousness, symptoms, severity and the availability, effectiveness and ease of use of vaccines, treatment and/or cure.

Conclusion While knowledge of individual STI infections varies, knowledge and awareness about Shigella was particularly low. MSM expressed a range of nuanced concerns related to individual STI sand STI testing and treatment. Fears influence and relate to each other e.g. considerable fear of a disfiguring infection may be reduced if the treatment is seen to be relatively easy, short and effective. MSM articulated a range of concerns which were subsequently used as categories in a self-completion survey to inform STI risk reduction, testing and treatment interventions.

\section{P4.91 MONITORING ADHERENCE TO PRE-EXPOSURE PROPHYLAXIS: DEVELOPING, USING AND OPTIMISING AN ONLINE TOOL FOR DAILY AND EVENT-DRIVEN REGIMEN}

${ }^{1}$ Reyniers Thijs, ${ }^{1}$ Bea Vuylsteke, ${ }^{2}$ Irith DE Baetselier, ${ }^{1}$ Christiana Nöstlinger, ${ }^{1}$ Marie Laga. ${ }^{1}$ Department of Public Health, Institute of Tropical Medicine, Antwerp, Belgium; ${ }^{2}$ Department of Clinical Sciences, Institute of Tropical Medicine, Antwerp, Belgium

\subsection{6/sextrans-2017-053264.586}

Introduction Oral Pre-Exposure prophylaxis (PrEP) with Truvada is a promising tool to reduce the number of HIV infections among men who have sex with men (MSM). With growing scientific interest in non-daily PrEP regimens (e.g. event-driven) assessing PrEP adherence has become more challenging. The aim was to develop, use and optimise an online tool to monitor PrEP adherence.

Methods In a first phase, an interdisciplinary team developed a secure web-based tool to collect data on adherence within a Belgian PrEP demonstration project (Be-PrEP-ared), involving 200 MSM using daily or event-driven PrEP or discontinuing PrEP use. The online diary was piloted before study implementation, and slightly adapted based on participant feedback. The use of the online diary tool by participants is closely monitored by study staff for completion. In a second phase, participative qualitative methods (i.e. co-creation sessions) were used to develop a smartphone application based on the diary aiming to support PrEP adherence. The efficacy of the adapted smartphone application is being tested.

Results The online diary is fully operational and being used by study participants of the PrEP demonstration project. Participants login into the secured platform on a daily base and fill in the number and timing of pills taken, whether they had sex that day and if so self-assessment of HIV risk self-assessment. The smartphone application is being finalised. Qualitative findings revealed that users prefer an application that provides scientific reliable information, is subtle and sensitive to privacy, can be used internationally, and has advanced 
automated features. Both online diary and smartphone application will be demonstrated.

Conclusion Web-based tools to monitor adherence to PrEP within trials are a novel and promising approach, with both advantages (eg. provides a large amount of data) and disadvantages (eg. limited validity). User-participation is an important pre-requisite to tailor such tools to target their needs.

\section{P4.92 START OF A SYNDEMIC BASED INTERVENTION TO FACILITATE CARE FOR MEN WHO HAVE SEX WITH MEN WITH HIGH RISK BEHAVIOUR: THE SYN.BAS.IN RANDOMISED CONTROLLED TRIAL}

${ }^{1}$ Roel Achterbergh, ${ }^{2}$ JJ Van Der Helm, ${ }^{3} \mathrm{~W}$ Van De Brink, ${ }^{4} \mathrm{AA}$ Matser, ${ }^{5} \mathrm{HJC}$ De Vries. ${ }^{1}$ Public Health Serice Amsterdam, Amsterdam, The Netherlands; ${ }^{2}$ Centre for Environmental Safety and Security, National Institute of Public Health and the Environment, Bilthoven, The Netherlands; ${ }^{3}$ Department of Psychiatry, Academic Medical Centre, Amsterdam, The Netherlands; ${ }^{4}$ Public Health Service Amsterdam, Amsterdam, The Netherlands; ${ }^{5}$ Department of Dermatology, Academic Medical Centre, Amsterdam, The Netherlands

\subsection{6/sextrans-2017-053264.587}

Introduction Men who have sex with men (MSM) constitute a risk group for sexual transmitted infections (STIs), including HIV. Despite counselling interventions, risk behaviour remains high. Syndemic theory holds that psychosocial problems often co-occur, interact and mutually reinforce each other, thereby increasing high risk behaviours and co-occurring diseases. Therefore, if co-occurring psychosocial problems were assessed and treated simultaneously, this might decrease high risk behaviour and disease.

Method An open label randomised controlled trial will be conducted among 150 MSM with high risk behaviour recruited from the STI clinic of Amsterdam. Inclusion criteria are: HIV negative MSM with two STI or PEP treatment in the last 24 months or HIV positive MSM with one STI in the last 24 months. All participants get questionnaires on the following syndemic domains: ADHD (ASRS), depression (HADS), anxiety disorder(HADS), alexithymia (TAS) and sex(SCS, Kalichman) and drug addiction(DUDIT and AUDIT). Participants in the control group receive standard care for one year: STI screenings every three months and motivational interviewing based counselling. Participants in the experimental group receive standard care plus additional questionnaires depending on baseline questionnaire scores followed by feedback and referral to a co-located mental health or addiction service in case of a positive indication according to the additional questionnaires. The primary outcome is help seeking behaviour for mental health problems and/or drug use problems. The secondary outcomes are STI incidence and changes in sexual risk behaviour (i.e. condom use, number of anal sex partners, drug use during sex).

Conclusion This study will provide information on syndemic domains among MSM who show high risk behaviour and on the effect of screening and referral on help seeking behaviour and health (behaviour) outcomes. Inclusion started in September 2016, on 1 December more than 60 MSM were included with this pace baseline data will be available in July 2017.

\section{P4.93 ARE RECTAL DOUCHING AND SHARING DOUCHING EQUIPMENT ASSOCIATED WITH ANORECTAL CHLAMYDIA AND GONORRHOEA? A CROSS-SECTIONAL STUDY AMONG MEN WHO HAVE SEX WITH MEN}

${ }^{1}$ Roel Achterbergh, ${ }^{2} \mathrm{JJ}$ Van Der Helm, 'W Van Den Boom, 'T Heijman, ${ }^{1} \mathrm{I} G$ Stolte, ${ }^{1} \mathrm{MS}$ Van Rooijen, ${ }^{3} \mathrm{HJC}$ De Vries. 'Public Health Serice Amsterdam, Amsterdam, The Netherlands; ${ }^{2}$ Centre for Environmental Safety and Security, National Institute of Public Health and the Environment, Bilthoven, The Netherlands; ${ }^{3}$ Department of Dermatology, Academic Medical Centre, Amsterdam, The Netherlands

\subsection{6/sextrans-2017-053264.588}

Introduction Men who have sex with men (MSM) are at high risk for anorectal chlamydia and gonorrhoea infections. Many MSM use rectal douches in preparation for sex, which might break down the mucosal barrier function and facilitate the acquisition of sexually transmitted infections (STI). We determined whether rectal douching or sharing douching equipment was associated with anorectal chlamydia and gonorrhoea.

Methods In a cross-sectional study among 994 MSM attending the STI outpatient clinic of Amsterdam between February and April 2011, data was collected on rectal douching, sexual behaviour, and STI. We used multivariable logistic regression analysis to determine the association between rectal douching, including sharing of douching equipment, and anorectal chlamydia and gonorrhoea for those at risk. We adjusted for other risk behaviour, i.e., condom use, number of partners, and HIV status.

Results Of 994 MSM, 46\% ( $=460)$ practised rectal douching, of whom 25\% ( $n=117)$ shared douching equipment. Median age was 39 years [interquartile range (IQR), 30-47], median number of sex partners in the six months prior to consult was five [IQR 3-10] and 289 (29.0\%) participants were HIVpositive. The prevalence of anorectal chlamydia and/or gonorrhoea for those at risk was 9.6\% $(\mathrm{n}=96)$. In multivariable analysis, $\mathrm{HIV}$ positivity $(\mathrm{aOR}=2.2,95 \% \mathrm{CI}=1.3-3.6)$, younger age $(\mathrm{aOR}=2.5, \mathrm{CI}=1.4-4.5$ for those $<35$ years compared to $\geq 45$ years), and more sexual partners $(\mathrm{aOR}=1.2 ; 95 \%$ $\mathrm{CI}=1.0-1.5$ for $1 \mathrm{log}$ increase) were significantly associated with anorectal STI. However, rectal douching or sharing douching equipment were not significantly associated with anorectal chlamydia and/or gonorrhoea $(p=0.726)$.

Conclusion Almost half of MSM used rectal douching and a quarter of these shared douching equipment. Though using douching equipment does not appear to contribute to anorectal chlamydia and gonorrhoea, STI prevalence remains high and prevention strategies like early testing and treatment remain of utmost importance.

\section{P4.94 STI RATES AMONG HOMELESS PERSONS IN THE U.S}

${ }^{1}$ Samantha P Williams, ${ }^{1}$ Alexandra E Caccamo, ${ }^{1}$ Rachel Kachur, ${ }^{2}$ Kenneth L Bryant. ${ }^{1}$ Division of STD Prevention, Centres for Disease Control and Prevention, Atlanta, USA; ${ }^{2}$ Division of Global HIV and TB, Centres for Disease Control and Prevention, Atlanta, USA

\subsection{6/sextrans-2017-053264.589}

Introduction Homelessness is a societal problem with public health implications. The U.S rate of homelessness is 17.7/ 10,000. Nightly, 550000 persons experience homelessness, $6.5 \%$ under the age of 18 years. Homelessness is associated with greater engagement in high-risk sexual behaviour. Less is known about sexually transmitted infections (STI) among the homeless. 